\title{
Comparison between Non-Inductive Plasma Current Start-Up Using ECRH with and without Fundamental Resonance on QUEST ${ }^{*}$
}

\author{
Hiroki MIURA, Kazuaki HANADA ${ }^{1)}$, Hideki ZUSHI ${ }^{1)}$, Kazuo NAKAMURA ${ }^{1)}$, \\ Akihide FUJISAWA $^{1)}$, Hiroshi IDEI ${ }^{1)}$, Yoshihiko NAGASHIMA ${ }^{1)}$, Makoto HASEGAWA ${ }^{1)}$, \\ Hisatoshi NAKASHIMA $^{1)}$, Shoji KAWASAKI ${ }^{1)}$, Aki HIGASHIJIMA ${ }^{1)}$, Osamu MITARAI ${ }^{2)}$, \\ Atsushi FUKUYAMA ${ }^{3)}$ and Yuichi TAKASE ${ }^{4)}$ \\ IGSES, Kyushu University, 6-1 Kasuga-koen, Kasuga 816-8580, Japan \\ ${ }^{1)}$ RIAM, Kyushu University, 6-1 Kasuga-koen, Kasuga 816-8580, Japan \\ ${ }^{2)}$ Tokai University, 9-1-1 Toroku, Higashi-ku, Kumamoto 862-8652, Japan \\ ${ }^{3)}$ RGPPE, Kyoto University, Nisikyo-ku, Kyoto 615-8540, Japan \\ ${ }^{4)}$ DCSE, University of Tokyo, 5-1-5 Kashiwa-no-ha, Kashiwa 277-8561, Japan
}

(Received 25 November 2014 / Accepted 21 April 2015)

\begin{abstract}
Comparison between non-inductive plasma current start-up using electron cyclotron resonance heating (ECRH) with and without fundamental (1st) resonance using 8.2-GHz microwave is experimentally performed on QUEST. Results of soft X-ray measurements in different energy ranges indicate significant difference in the number of current-carrying electrons with and without fundamental resonance. Numerical calculations show effective extendibility of electron energy in ECRH of fundamental resonance.
\end{abstract}

(C) 2015 The Japan Society of Plasma Science and Nuclear Fusion Research

Keywords: spherical tokamak, plasma current start-up, electron cyclotron resonance heating, current-carrying electron, soft X-ray

DOI: $10.1585 / \mathrm{pfr} .10 .3402066$

\section{Introduction}

Because of their higher compactness with low aspect ratio $(A=R / a: R$ the major radius and $a$ the minor radius), spherical tokamaks (STs) are the leading candidates for economically efficient fusion reactors. STs are capable of stably confining high- $\beta$ plasmas ( $\beta$ denoting the ratio of plasma pressure to magnetic pressure) [1]. In conventional tokamaks, Ohmic heating $(\mathrm{OH})$ using a toroidal electric field inductively-induced by the time variation of the current through the center solenoid (CS) coil is the main method to generate and control plasma current. Because space is limited in the high-field side of a ST, non-inductive plasma current start-up is an indispensable technique. Experiments of non-inductive plasma current start-up using electron cyclotron resonance heating (ECRH) have been actively performed in ST such as LATE [2], MAST [3], TST-2 [4] and QUEST [5,6]. In the experiments, the combined effect of fundamental (1st) and second harmonic (2nd) ECRH provides a significant plasma heating [2, 5], and it is also reported the energetic electrons confined in an open magnetic surface plays an essential role in plasma current start-up [5,6]. In conventional tokamaks, plasma production with 2 nd ECRH has been reported [7,8] and a

author's e-mail: miura@triam.kyushu-u.ac.jp

*) This article is based on the presentation at the 24th International Toki Conference (ITC24). plausible explanation has been developed $[9,10]$. Nevertheless, plasma current start-up without 1st ECRH has not been reported yet.

Our challenge is to make a directly-compared survey of the effectiveness of ECRH in plasma current start-up with and without 1 st ECRH.

The experimental setup is described in Section 2. The results of experiments are presented in Section 3. The numerical calculation on the efficiency in energy absorption from the electric field to electrons is presented in Section 4. A summary is presented in Section 5.

\section{Experimental Apparatuses}

Experiments have been performed on QUEST, which is a middle-sized ST with $R=0.64 \mathrm{~m}, a=0.40 \mathrm{~m}$, and toroidal magnetic field $B_{\mathrm{T}}<0.25 \mathrm{~T}$, and electron cyclotron wave with frequency of $8.2 \mathrm{GHz}, \mathrm{O}$-mode is launched on the mid-plane of the vessel as the main heating source. The vacuum magnetic field configuration in the experiments has a positive n-index and there is no magnetic-field null inside of the vacuum vessel (Fig. 1). The magnetic configuration plays an essential role in confining the energetic electrons $[5,6]$ and gives successful plasma current start-up with 1 st ECRH of $8.2 \mathrm{GHz}[5,6]$. In the set of experiments for the study, the same vertical field is adopted unless otherwise stated. 

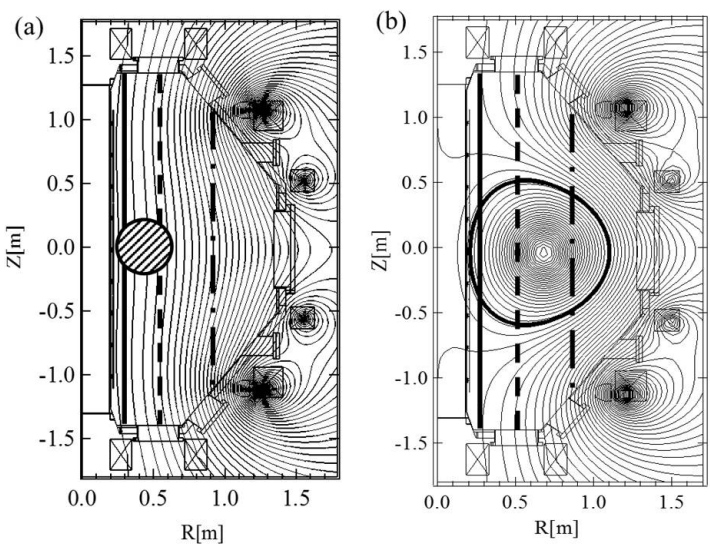

Fig. 1 Typical contours of the poloidal flux (a) without and (b) with plasma current (\#24498 $2.2 \mathrm{~s}$ ). Outlines of the vacuum vessel and the poloidal coils are also shown. The vertical lines indicates the position of 1 st (solid), 2nd (dashed) and 3rd (dashed-dotted) ECR layers. A solid circle in Fig. 1 (a) indicates the viewing area of the 2DSXR camera which are $26-62 \mathrm{~cm}$ on toroidal direction and $-18-+18 \mathrm{~cm}$ on vertical direction.
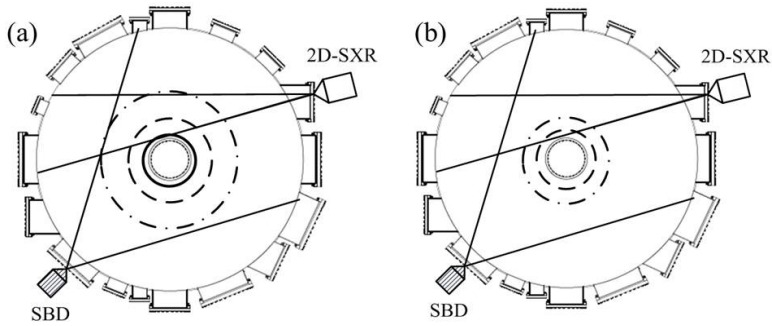

Fig. 2 Top cross-sectional views of the vessel and visible area of both the SBD and the 2D-SXR detectors. (a): Loci of the 1st (solid), the 2nd (dashed) and 3rd (dashed-dotted) ECR layers of Fig. 1 (a) are drawn with $R=29 \mathrm{~cm}, 58 \mathrm{~cm}$ and $87 \mathrm{~cm}$, respectively. (b): Loci of the 2 nd (dashed) and 3rd (dashed-dotted) ECR layers are given with $R=$ $31 \mathrm{~cm}$ and $48 \mathrm{~cm}$, respectively.

Two types of soft X-ray (SXR) measurement are used in the experiments. One is a surface barrier diode (SBD) and another is a two-dimensional SXR (2D-SXR) camera. The detectable energy range of the SBD is from $10 \mathrm{eV}$ to $80 \mathrm{eV}$. The SBD is located on the mid-plane to monitor the whole plasma. The energy sensitivity of the 2D-SXR camera was regulated in the range of $60-80 \mathrm{eV}$ to detect the production of current-carrying electrons. The viewing area of the camera can be illustrated in Fig. 1 and Fig. 2.

\section{Experimental Results}

Typical waveforms for the start-up experiments with and without 1st ECRH are shown in Fig. 3. Plasma production and plasma current start-up take place almost simultaneously as observed in the signals of $\mathrm{H} \alpha$, SXR and plasma current. Plasma currents of $8 \mathrm{kA}$ and $3 \mathrm{kA}$ were achieved with and without 1 st ECRH, respectively. To improve the
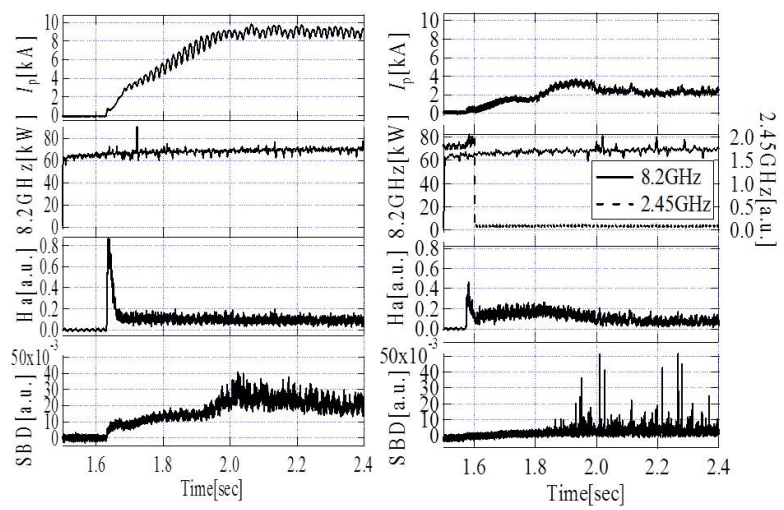

Fig. 3 Typical waveforms of plasma current, RF power, $\mathrm{H} \alpha$ intensity and SXR intensity detected by the SBD in 1st ECRH plasma current start-up (\#24498) (left) and those with 2nd ECRH (\#25673) (right). The location of the ECR layers in each plasma is illustrated in Fig. 2.

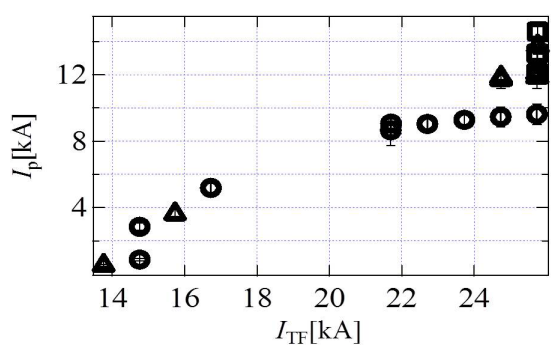

Fig. 4 The toroidal field coil current dependence on the generated plasma current. Data below $I_{\mathrm{TF}}=17 \mathrm{kA}$ (without 1 st ECRH) in the range of $68-70 \mathrm{~kW}$ and data above $I_{\mathrm{TF}}$ $=21 \mathrm{kA}$ (with 1st ECRH) in the range of $65-70 \mathrm{~kW}$ are plotted. $I_{\mathrm{TF}}=26.6 \mathrm{kA}$ corresponds to the major radius of 1 st ECR $\left(R_{1 \mathrm{st} \mathrm{ECR}}\right)=29 \mathrm{~cm}, R_{2 \text { nd ECR }}=58 \mathrm{~cm}$ and $R_{3 \mathrm{rd} \mathrm{ECR}}$ $=87 \mathrm{~cm}$. For $I_{\mathrm{TF}}=18-21 \mathrm{kA}$, as the $1 \mathrm{st}$ ECR is located at $R_{\mathrm{ECR}}=20-23 \mathrm{~cm}$, where the gap is between the inner vessel and the inner protector, and the operation is prohibited. The three symbols represent data at ECR from different vertical field strengths of $2.6 \mathrm{mT}$ (circles), $2.7 \mathrm{mT}$ (triangles), and $3.2 \mathrm{mT}$ (squares).

reproducibility of the plasma production, the RF power for the $2.45 \mathrm{GHz}$ is applied just before the injection of the RF power for $8.2 \mathrm{GHz}$. Indeed, it should be noted (Fig. 3) that the application of $2.45 \mathrm{GHz}$ cannot produce plasma current except pressure driven current. ( $\sim 0.5 \mathrm{kA})$. To drive further plasma current, it is necessary to produce energetic electrons which have the capability to drive plasma current due to the intrinsic toroidal asymmetric confinement in co and counter drift direction [4-6]. As plasma current starts up just after the termination of the injection of $2.45 \mathrm{GHz}$, the pre-injection of the $2.45 \mathrm{GHz}$ RF does not play an essential role in the plasma current start-up. The generated plasma current without 1st ECRH is significantly lower than that with 1st ECRH even in the nearly identical poloidal magnetic configuration. From the ECR location dependence (Fig. 4), higher toroidal fields can provide higher plasma 


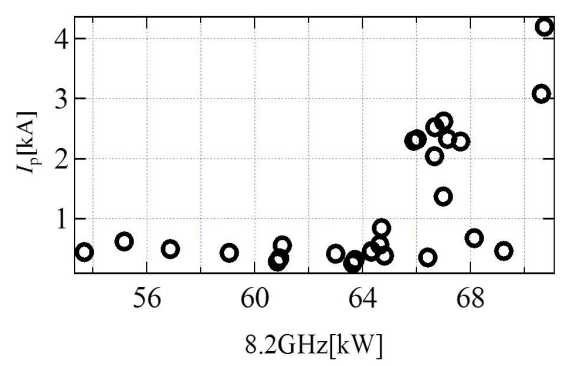

Fig. 5 Plasma current vs RF power without 1st ECRH. The 2nd ECR is located in the range of $R_{2 \text { nd ECR }}=30-37 \mathrm{~cm}$, and $I_{\mathrm{TF}}=14-17 \mathrm{kA}$
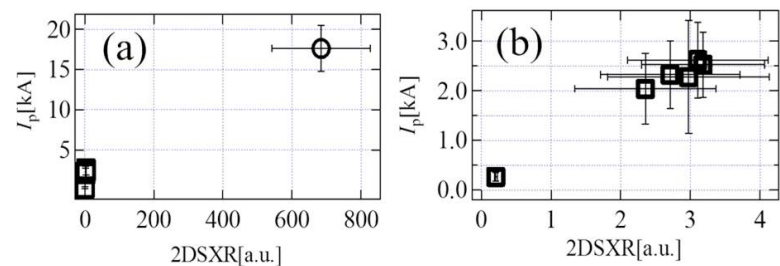

Fig. 6 (a) The relationship between SXR intensity as measured by the 2D-SXR camera and plasma current is the same that obtained with the SBD. Circles and squares show the data with 1st ECRH (\#25688) and without 1st ECRH (\#25669-25677), respectively. (b) Enlarged view of data without 1st ECRH.

currents in all cases. Plasma current with 1st ECRH is usually higher than that without 1st ECRH. The generated plasma current also depends on the injected RF power (Fig. 5). Breakdown and production of pressure driven current occur during injection with $\mathrm{RF}$ power below $65 \mathrm{~kW}$, but without initiating any other plasma current. Plasma current flows without 1st ECRH when the RF power is above $65 \mathrm{~kW}$. Higher plasma current is generated by increasing the RF power. The total intensity of SXRs detected with the 2D-SXR camera is plotted as a function of the plasma current (Fig. 6). Values have an apparent positive correlation with plasma current and this indicates that energetic electrons drive plasma current. It has already reported that current-carrying electrons on QUEST has the energy in the range of $100 \mathrm{eV}$ to several $\mathrm{keV}$ during the plasma current start-up phase [11]. This correlation can be obtained in the previous observations on vertical-field dependence at plasma current start-up with 1st ECRH [12]. The relation between the ratio of SBD to $\mathrm{H} \alpha$ intensities and plasma current is presented in Fig. 7. This ratio is favored as a monitor of the number of energetic electrons. A significant difference in the ratio between with and without 1st ECRH is obtained. Only the toroidal field strength is adjusted to switch harmonics patterns in the vacuum vessel. This strongly indicates that the difference in heating mechanism gives rise to the difference in the number of energetic electrons and plasma current. One should be considered how the number of energetic electron in higher harmonics

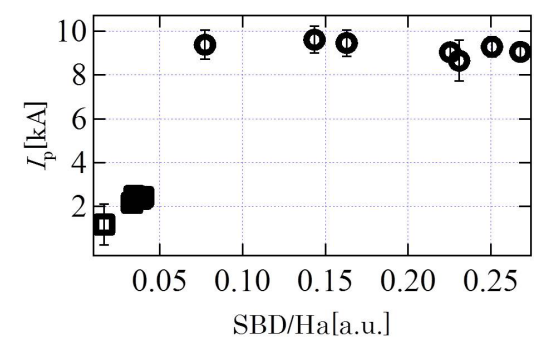

Fig. 7 The ratio between the SXR detected by the SBD and $\mathrm{H} \alpha$ is plotted as a function of plasma current. Circles and squares show data with 1st ECRH (\#24495-24502) and without 1st ECRH (\#25673-25677), respectively.

representing 2nd ECRH is significantly less than that in 1st ECRH.

\section{Numerical Calculation of Electron Energy Absorbed from Electric Field}

The electron energy is sourced from the electrical field of the high-frequency wave when electrons pass through the ECR layer is calculated using a model given in $[9,10]$. The phase difference between the particle gyromotion and the wave electric field as $\Psi=n \psi-\omega t$ and the dimensionless variable $\Gamma=\gamma-1$ are defined, where, $n, \psi, \omega, \gamma$ are the harmonic number, the gyro-phase of electrons, the wave angular frequency, and relativistic factor of electron, respectively. $\Gamma m c^{2}$ is the kinetic energy of electrons and $\Gamma$ is the normalized kinetic energy of electrons and intrinsically $\Gamma \ll 1$. The basic equations of $\Gamma$ and $\Psi$ are derived from the Lorentz equation for electrons including relativistic effect in the electric field of the microwave beam under the assumption of $k \rho_{\mathrm{L}} \ll 1$, where $k$, and $\rho_{\mathrm{L}}$ are the wave number, Larmor radius of electron, respectively.

$$
\begin{array}{rlrl}
\frac{\mathrm{d} \Psi}{\mathrm{d} t} & =-\Gamma \omega+\frac{1}{2 \sqrt{2}} \varepsilon \omega \Gamma^{-\frac{1}{2}} \sin \Psi \\
& =-\Gamma \omega+\varepsilon \omega \cos \Psi & \\
\frac{\mathrm{d} \Gamma}{\mathrm{d} t} & =-\frac{\varepsilon \omega}{\sqrt{2}} \Gamma^{\frac{1}{2}} \cos \Psi & & (\text { For } n=1) \\
& =\varepsilon \omega \Gamma \sin \Psi & & (\text { For } n=2) .
\end{array}
$$

(For $n=1)$

(For $n=2$ ),

Here $\varepsilon \equiv e \boldsymbol{E} / m c \omega$ is the ratio of the momentum change of particles to $m c$ during one wave period. The wave frequency is set at $8.2 \mathrm{GHz}$, injection RF power is $60 \mathrm{~kW}$, and the half-width of electrical field is set to $20 \mathrm{~cm}$ at $1 \mathrm{st}$ and 2nd ECR. The time evolutions for the energy transfer to electrons in 2nd ECR is presented in Fig. 8 (a). The initial kinetic energy of the injected electron is $\Gamma=1 \times 10^{-6}$, which corresponds to approximately room temperature. This calculation indicates resonance electrons in the strong electric field can be accelerated immediately, but the most of the transferred energy is released when the electron moves out of the strong electric field. Nevertheless, some 

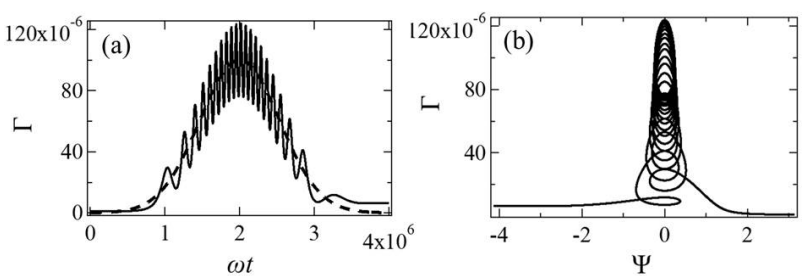

Fig. 8 (a) Variation of $\Gamma$ with initial energy, which is set to $1 \times$ $10^{-6}$ for $\omega t$ of 2 nd ECR. $\Gamma$ oscillates when electrons pass through the RF electrical field. The dashed line gives its strength. (b) Variation of $\Gamma$ with initial energy, which is $1 \times 10^{-6}$ for $\Psi$ of 2 nd ECR.

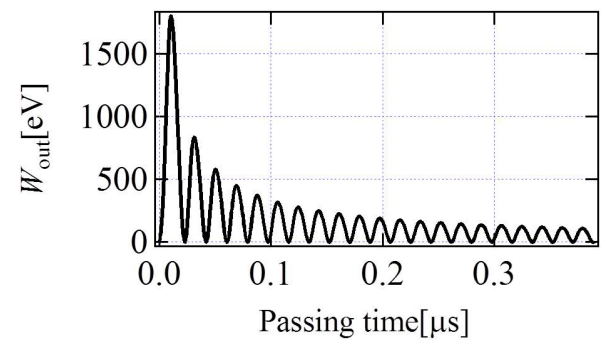

Fig. 9 Absorbed energy at zero electric field as a function of elapsed time through the high-frequency electric field at 1 st ECR.

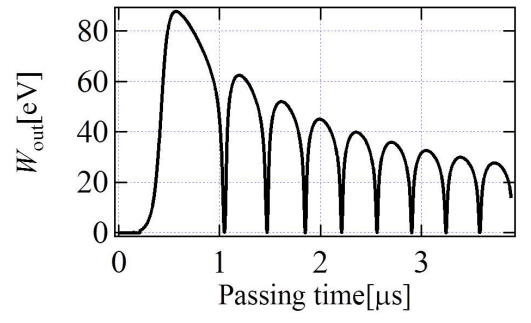

Fig. 10 Absorbed energy at zero electric field as a function of elapsed time through the high-frequency electric field at 2nd ECR.

of the transferred energy is retained. The mechanism is easy to understand by referring to the $\Gamma-\Psi$ mapping (Fig. 8 (b)), where $\Psi$ is the phase difference associated with the electron cyclotron motion and the electrical field frequency multiplied by the harmonic number. In strong electric fields, electrons trace out ellipses, the size of which can be determined by the strength of electric field on the $\Gamma-\Psi$ map. $\Gamma$ is an invariant of motion when the variation of electric field is significantly slower than the rotation frequency for the ellipse. Breaking of the $\Gamma$ invariance is the only way to transfer energy to the electron and is required in varying the electron-affected electric field strength as fast as possible. The value of $\Gamma$ for the ellipse at the same electric field in 2nd ECR is significantly lower than that in 1st ECR. Moreover the rotation frequency strongly depends on the harmonic number and the frequency at 2 nd ECR is clearly lower than that in the 1st ECR. The cal- culation finds that 3rd ECR is inherently more inefficient. Let $W_{\text {out }}$ denote the electron energy at zero electric field when an electron is passing through the electric-field at ECR. From $W_{\text {out }}$ for 1st and 2nd ECR given in Figs. 9 and 10 respectively, their maximum energies are $1.8 \mathrm{keV}$ and $87 \mathrm{eV}$. Clearly, the maximum energy at 2nd ECR is much lower than that at 1st ECR. 1st ECRH are possible to be made current-carrying electrons, but 2nd ECRH is difficult. It is thought that the effects of synergistic and stochastic heating are necessary to produce current-carrying electrons without 1st ECRH. In fact, stochastic heating is working well to accelerate electron up to $8 \mathrm{keV}$ at 1 st ECRH [13] in the same RF power, although the efficiency is not so high.

\section{Summary}

Plasma current start-up is achieved without 1st ECRH using $8.2-\mathrm{GHz}$ microwave. Plasma current tends to be higher with increasing RF power and toroidal field, although plasma current without 1st ECRH is always less than that with 1st ECRH. The number of current-carrying electrons with 1st ECRH is higher than that without 1st ECRH because of the total intensity of SXRs detected with the 2D-SXR camera. The acceleration of electrons in 2nd ECR is slower and electrons in 2nd ECR receives less energy from the RF electrical field from the numerical calculations. It is difficult to generate current-carrying electrons in 2nd ECR. It is understood that 2nd ECR is inherently inefficient to start up plasma current. We confirm that our results from calculations were qualitatively consistent with experimental results.

\section{Acknowledgments}

This work was partially supported by JSPS KAKENHI $(24226020,24656559)$ and was performed with the support and under the auspices of the NIFS Collaboration Research program (NIFS13KUTR085, NIFS13KUTR093). This work was supported in part by the Collaborative Research Program of Research Institute for Applied Mechanics, Kyushu University.

[1] Y.-K.M. Peng, Phys. Plasmas 7, 1681 (2000).

[2] M. Uchida et al., Phys. Rev. Lett. 104, 065001 (2010).

[3] V.F. Shevchenco et al., Nucl. Fusion 50, 022004 (2010).

[4] A. Ejiri et al., Nucl. Fusion 47, 403 (2007).

[5] M. Ishiguro et al., Phys. Plasmas 19, 062508 (2012).

[6] S. Tashima et al., Nucl. Fusion 54, 023010 (2014).

[7] Y.S. Bae et al., Nucl. Fusion 49, 022001 (2009).

[8] G.L. Jackson et al., Nucl. Fusion 47, 257 (2007).

[9] J. Seol et al., Phys. Plasmas 16, 052512 (2009).

[10] J. Seol et al., Nucl. Fusion 50, 105008 (2010).

[11] M. Ishiguro et al., Rev. Sci. Instrum. 82, 113509 (2011).

[12] K. Hanada et al., Plasma Sci. Technol. 13, 307 (2011).

[13] M. Ishiguro, Study on Non-inductive Current Start-up in Spherical Tokamaks based on Direct Measurement of Energetic Electrons, Kyushu University, Doctoral Thesis (2011). 